Please do not remove this page

RMIT

UNIVERSITY

\title{
FBG-based optical interface to support a multisector antenna in a spectrally efficient fiber radio system
}

Marra, C; Nirmalathas, Ampalavanapillai; Lim, C.; Attygalle, M; Novak, D; Ashton, B; Poladian, L https://researchrepository.rmit.edu.au/esploro/outputs/9921857840601341/filesAndLinks?institution=61RMIT_INST\&index=null

Marra, C., Nirmalathas, A., Lim, C., Attygalle, M., Novak, D., Ashton, B., Poladian, L., Rowe, W., Wang, T., \& Besley, J. A. (2004). FBG-based optical interface to support a multisector antenna in a spectrally efficient fiber radio system. IEEE Photonics Technology Letters, 16, 254-256.

https://doi.org/10.1109/LPT.2003.818952

Published Version: https://doi.org/10.1109/LPT.2003.818952

Repository homepage: https://researchrepository.rmit.edu.au

(c) 2004 IEEE. Personal use of this material is permitted. However, permission to reprint/republish this material for advertising or promotional purposes or for creating new collective works for resale or redistribution to servers or lists, or to reuse any copyrighted component of this work in other works must be obtained from the IEEE.

Downloaded On 2023/04/26 21:12:37 +1000 


\title{
FBG-Based Optical Interface to Support a Multisector Antenna in a Spectrally Efficient Fiber Radio System
}

\author{
Charlotte Marra, A. Nirmalathas, Member, IEEE, C. Lim, M. Attygalle, D. Novak, B. Ashton, L. Poladian, \\ W. S. T. Rowe, T. Wang, and J. A. Besley
}

\begin{abstract}
We propose and demonstrate a fiber Bragg grating (FBG)-based optical interface for use in a spectrally efficient fiber-radio network with multisector antennas. The system has the novel feature of being specifically developed for use in existing wavelength-division-multiplexed network infrastructures. The proposed scheme supports transport of a remote local oscillator (LO) and three subcarrier multiplexed data channels, destined for different antenna sectors, using a single wavelength. The composite signal was contained within a $25-\mathrm{GHz}$ band, selected via a 25-GHz dispersion-flattened FBG. Recovery of the LO and data channels is performed via optical filtering, using either a novel single grating incorporating multiple phase shifts or multiple narrow bandwidth gratings. Our measurements show that all channels within the $25-\mathrm{GHz}$ band are successfully recovered with less than 2-dB optical power penalty between channels. The use of the 25-GHz grating exhibits an improvement in sensitivity of $3 \mathrm{~dB}$ for all data channels.
\end{abstract}

Index Terms-Gratings, millimeter-wave (mm-wave) radio communication, optical fiber communication, optical filters, subcarrier multiplexing (SCM).

\section{INTRODUCTION}

$\mathbf{T}$ HE development of wireless communication systems operating at millimeter-wave (mm-wave) frequencies will assist in the delivery of future broad-band services with data rates $>1 \mathrm{~Gb} / \mathrm{s}$ [1]. To accelerate a commercial deployment of fiber-radio architectures, existing wavelength-division-multiplexed (WDM) broad-band optical network infrastructures based on ITU grid spacings must be efficiently utilized. While transporting the radio signal over fiber in a mm-wave format has the advantage of simplifying the antenna base-station (BS) architecture, optical channel spacings in excess of $60 \mathrm{GHz}$ may be required [2], thereby limiting the transport scheme to one mm-wave channel per WDM wavelength. In particular, for a fiber feed network incorporating BSs with multiple sectorized antenna interfaces, each sector needs to be fed by separate mm-wave signals, requiring multiple WDM channels to provide the full interconnection to the central office $(\mathrm{CO})$

Manuscript received Febrauary 19, 2003; revised July 24, 2003.

C. Marra, A. Nirmalathas, C. Lim, and M. Attygalle are with the Australian Photonics Cooperative Research Centre, Photonics Research Laboratory, Department of Electrical and Electronic Engineering, The University of Melbourne, Melbourne, VIC 3010, Australia (e-mail: cnmarra@ee.mu.oz.au).

D. Novak is with the Corvis Corporation, Columbia, MD 21046 USA.

B. Ashton and L. Poladian are with the Optical Fiber Technology Centre, APCRC, University of Sydney, Sydney, NSW 1430, Australia.

W. S. T. Rowe is with the Australian Photonics CRC, School of Electrical and Computer Engineering, RMIT University, Melbourne, 3001 Australia.

T. Wang is with the JDS Uniphase, Sydney, NSW 2113, Australia.

J. A. Besley is with the Department of Physics, University of Sydney, NSW

2006, Australia.

Digital Object Identifier 10.1109/LPT.2003.818952

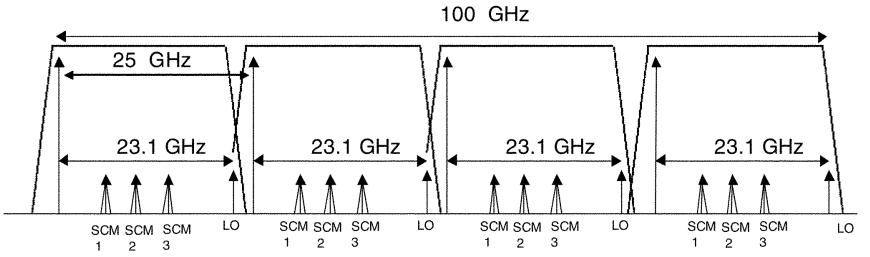

Fig. 1. Wavelength allocation scheme for a spectrally efficient WDM fiber-radio network incorporating simultaneous SCM and remote LO delivery.

which is spectrally inefficient. To improve the optical spectral efficiency, the radio signal feeding each sector can be transported as a low frequency subcarrier multiplexed (SCM) signal on a single WDM wavelength. However, this approach requires a mm-wave local oscillator (LO) at the BS for frequency upconversion to mm-wave frequencies, increasing both $\mathrm{BS}$ complexity and cost. To overcome this problem, a number of techniques involving the simultaneous transmission of the LO and data signals from the $\mathrm{CO}$ to the BSs have been proposed. These techniques include the transmission of the LO at a $\mathrm{mm}$-wave frequency [3], or as a lower frequency harmonic of the LO [4], [5]. However, such schemes have drawbacks for application in a multisector fiber radio network, such as spectral inefficiency, the potential to generate higher order side modes or the requirement for complex electronics in the BS [3]-[5].

In this letter, we propose and demonstrate a fiber Bragg grating (FBG)-based interface for a fiber-radio network which utilizes remote optical LO delivery together with multiple sector signal transport, contained within a $25-\mathrm{GHz}$ frequency band. The proposed architecture offers an improved optical spectral efficiency and overcomes disadvantages associated with other LO delivery and recovery schemes. The interface incorporates novel FBG designs and simple narrow-band optical filtering not previously demonstrated to recover an LO and data signals.

\section{DESIGN OF THE FBG FOR LO AND INTERMEDIATE FREQUENCY (IF) FILTERING}

To improve the spectral efficiency of multisector fiber-radio networks, three data channels were assigned to each access network wavelength. The subcarrier frequencies $(7.1,10.22$, and $13 \mathrm{GHz}$ ) and LO signal frequency of $23.1 \mathrm{GHz}$ were chosen such that the electrical bandwidth lies within a $25-\mathrm{GHz}$ band [7]. The electrical signal then modulated the carrier wavelength using optical single sideband with carrier $(\mathrm{OSSB}+\mathrm{C})$ modulation which allows the LO and SCM signals to be contained within the $25-\mathrm{GHz}$ band and reduces fiber chromatic dispersion effects [8]. Fig. 1 depicts the allocation of the LO and SCM channels with their corresponding optical carriers as can 


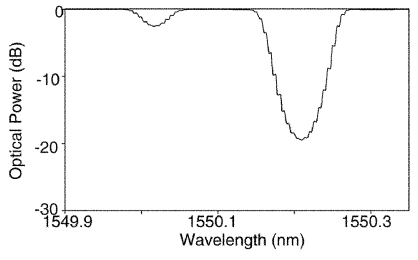

(a)

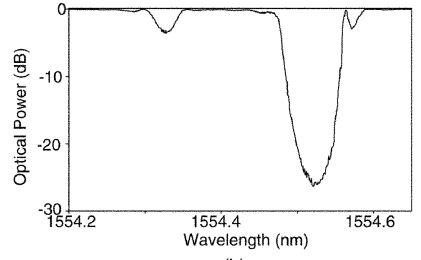

(b)
Fig. 2. Measured transmission responses of FBG designs for LO recovery (a) Two concatenated FBGs and (b) single novel FBG structure with eight phase shifts of $64^{\circ}$.

be implemented in existing access network infrastructures. Successful recovery of the LO and SCM channels contained within a signal band is achieved at the BS using narrow-band optical filtering techniques implemented via novel FBGs. This detection process requires a portion of the optical carrier to be filtered with the LO modulation sideband, with the remaining carrier power detected with the SCM channels. To achieve the reflectivity profiles necessary for this filtering, two different FBG designs were implemented, both reflecting half the carrier and all of the LO, while passing the SCM signals unaffected. The first design consisted of a concatenation of two separate FBGs with bandwidths of less than $10 \mathrm{GHz}$ and transmission strengths of -2.5 and $-18 \mathrm{~dB}$, as shown in Fig. 2(a). The second FBG was designed to have an improved transmission notch rolloff and depth, when compared with the two grating cascade. Fig. 2(b) shows the transmission response of this novel FBG design which consists of a single grating structure incorporating a total of eight strategically located phase shifts of $64^{\circ}$. A $25-\mathrm{GHz}$ dispersion-flattened grating was also fabricated for the filtering of the carrier, LO, and three SCM signals within a $25-\mathrm{GHz}$ band to feed the appropriate multisector BS. Since the focus of this work was the implementation of the FBG-based interface and recovery of the LO and data signals via narrow-band optical filtering, transmission of a single wavelength $25-\mathrm{GHz}$ band was investigated. Future work will address the transmission of multiple wavelengths in the fiber-radio network.

\section{EXPERIMENT}

Fig. 3 shows the experimental setup. At the CO, the three SCM signals were modulated in binary phase-shift keying format via a $60-\mathrm{Mb} / \mathrm{s} 2^{15}-1$ pseudorandom binary sequence data signal, amplified, and combined with the LO signal. The resulting radio-frequency (RF) signal was then applied to a dual-electrode Mach-Zehnder modulator via a hybrid coupler and used to modulate, in OSSB $+\mathrm{C}$ format, the output from a tunable laser source. The signal was amplified by an erbium-doped fiber amplifier to compensate for the $10-\mathrm{km}$ single-mode-fiber (SMF) link loss and input into a bandpass filter (BPF) to remove amplified spontaneous emission (ASE) noise. It was then incident on the $F B G(s)$ via a circulator, with the transmitted and reflected spectrums from the two concatenated gratings as inserts in Fig. 3. Both the reflected LO and transmitted SCM signals were detected (in the BS) via high-speed photodetectors (PDs). A variable optical attenuator was used in the SCM optical arm to vary the received optical power, which was measured at the input to the SCM PD. The RF signal was then electrically filtered to separate the SCMs, amplified, and upconverted using the recovered 23.1-GHz LO to produce a frequency at $30.2,33.32$, or $36.1 \mathrm{GHz}$. Further upconversion of these signals to the $37-\mathrm{GHz}$ range now requires the operating frequency of the LO at the BS to be less than $7 \mathrm{GHz}$. This BS LO is, thus, less expensive and more readily available than the mm-wave LO that would be required at the $\mathrm{BS}$ if there was no 23.1-GHz remote LO delivery. Finally, the 36-, 37-, or 38-GHz mm-wave signals were passed through a $37-\mathrm{GHz} \mathrm{BPF}$, transmitted to the customer unit and downconverted to $2.5 \mathrm{GHz}$, fed into an electronic phase lock loop and the recovered data input into the bit-error-rate (BER) testset for BER measurements.

\section{RESULTS AND DISCUSSION}

Fig. 4(a) and (b) shows the measured BERs for the $60-\mathrm{Mb} / \mathrm{s}$ data recovered from each SCM signal after optical filtering using the concatenation of two FBGs or the novel single phase-shifted FBG, respectively. Six curves are shown in each figure, corresponding to recovery of the 7.1-, 10.22-, or $13-\mathrm{GHz}$ SCM signals with or without adjacent SCM frequencies also transmitted. The results in Fig. 4 show an optical power penalty spread of only $2 \mathrm{~dB}$ at a BER of $10^{-9}$ between all three SCM channels, demonstrating the ability to successfully utilize narrow-band optical filtering techniques for remote LO recovery, in a spectrally efficient fiber-radio network. In particular, the results show how the novel phase-shifted grating offers comparable performance to a grating cascade. The small difference in the slope of the BER curves for the $13-\mathrm{GHz} \mathrm{SCM}$ is due to the different phase response of the 13-GHz RF filter. The 7.1-GHz SCM channel exhibits the best performance using both filtering schemes, although the 10.22and $13-\mathrm{GHz}$ SCM channels show a change in sensitivity when using the phase-shifted grating. This can be attributed to small dispersion differences between the two FBG designs and some experimental error, however, the power penalty spread between all three SCM channels remained the same with either filtering scheme. In addition, the observed power penalty was less than $0.25 \mathrm{~dB}$ in both Fig. 4(a) and (b), when each SCM is recovered either with or without the adjacent SCM channels transmitted. This very small power penalty is within experimental error, illustrating how multiple SCM data channels and a remote LO can be simultaneously transmitted and recovered with minimal degradation and distortion arising from adjacent channels.

To further demonstrate the successful application of FBGs as narrow-band optical filters in a fiber-radio architecture with remote LO delivery, the $25-\mathrm{GHz}$ dispersion-flattened grating and second circulator were inserted after the $10 \mathrm{~km}$ of SMF. Fig. 5 shows the measured BERs for data recovery from each SCM signal, via filtering with the phase-shifted FBG, when all SCM channels are transmitted. A comparison with Fig. 4(b) shows that the power penalty spread remains $2 \mathrm{~dB}$ at a BER of $10^{-9}$ with the BER trends between data channels maintained. The use of the 25-GHz FBG has the advantage of improving the optical sensitivity for all channels by $3 \mathrm{~dB}$ due to the additional filtering of ASE noise it provides. It should be noted that while the $60-\mathrm{Mb} / \mathrm{s}$ data rate demonstrated in the experiment was determined by RF component availability, the proposed architecture can be easily extended to higher bit rates with no expected change to power penalty spreads. 


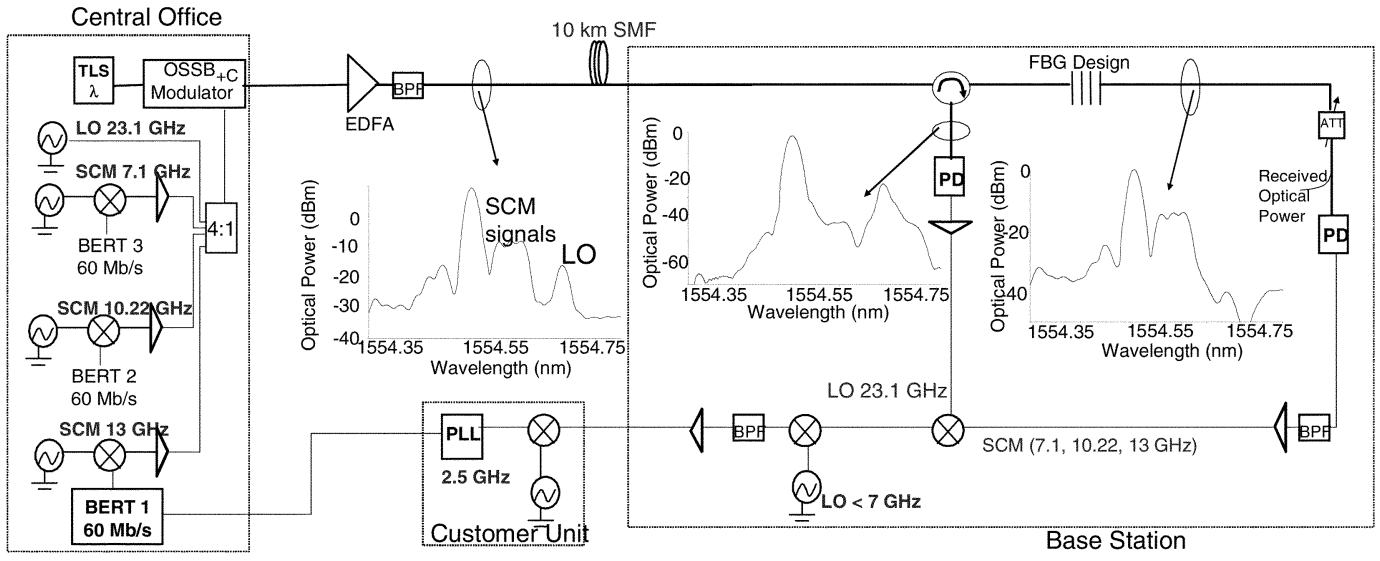

Fig. 3. Experimental setup to demonstrate simultaneous delivery and recovery of a remote LO and three IF SCM data signals, in a 25-GHz band, using novel FBG optical filtering techniques.

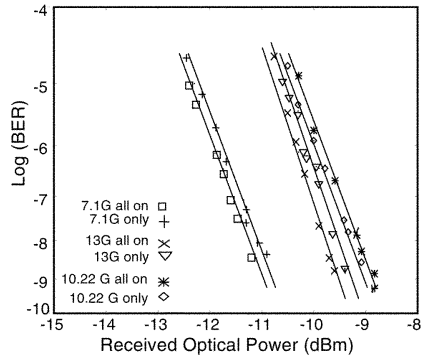

(a)

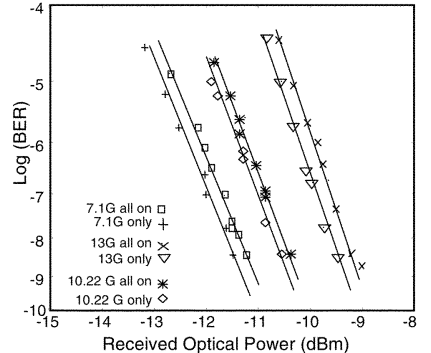

(b)
Fig. 4. Measured BER performance for three SCM channels, using remote LO delivery and optical filtering via (a) two cascaded FBGs or (b) a single eight-phase-shifted FBG.

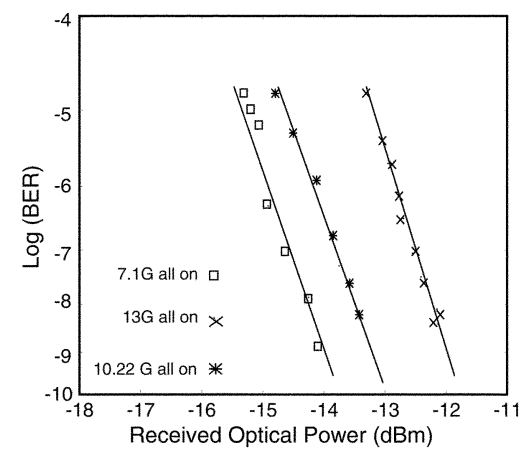

Fig. 5. Measured BER performance for three SCM channels, using remote LO delivery and optical filtering via a $25-\mathrm{GHz}$ dispersion-flattened FBG in conjunction with an eight-phase-shifted FBG.

\section{CONCLUSION}

An FBG-based interface has been proposed for use in a spectrally efficient fiber-radio mm-wave network. The network utilizes remote LO delivery together with multisector SCM data transmission, with the narrow-band optical filtering techniques overcoming disadvantages associated with other proposed remote LO delivery and recovery schemes. The performance of two different types of FBG designs, one incorporating multiple phase shifts, was compared in an experimental system application. The demonstration investigated the transmission and recovery of an LO and three SCM data signals located within a $25-\mathrm{GHz}$ band using a single wavelength. Successful recovery of the LO and all SCM channels was achieved with a power penalty spread of less than $2 \mathrm{~dB}$ for all channels, after transmission over $10 \mathrm{~km}$ of fiber, for both types of FBG designs. The additional use of a $25-\mathrm{GHz}$ grating filter does not degrade individual data channel performance and assists in improving the optical sensitivity. The demonstrated optical filtering technique for LO recovery is simple and inexpensive, ultimately reducing BS complexity and cost, with the simultaneous transmission of three data channels allowing for multisector capability in the network. These advantages, combined with the improved spectral efficiency, are key components for accelerating the initial deployment of mm-wave fiber-radio networks in existing WDM infrastructures.

\section{REFERENCES}

[1] G. Smith, D. Novak, and C. Lim, "A millimeter-wave full duplex fiber-radio star-tree architecture incorporating WDM and SCM," IEEE Photon. Technol. Lett., vol. 10, pp. 1650-1652, Nov. 1998.

[2] K. Kitayama, T. Kuri, K. Onohara, T. Kamisaka, and K. Murashima, "Dispersion effects of FBG filter and optical SSB filtering in DWDM millimeter-wave fiber-radio systems," J. Lightwave Technol., vol. 20, pp. 1397-1407, Aug. 2002.

[3] T. Kuri, K. Kitayama, and Y. Ogawa, "A novel fiber-optic millimeter-wave uplink incorporating $60 \mathrm{GHz}$-band photonic downconversion with remotely fed optical pilot tone using an electoabsorption modulator," in Proc. Int. Topical Meet. Microwave Photonics, Princeton, NJ, 1998, pp. 17-20.

[4] C. Lim, A. Nirmalathas, D. Novak, R. Waterhouse, and G. Yaffe, "Millimeter-wave broad-band fiber-wireless system incorporating baseband data transmission over fiber and remote LO delivery," $J$. Lightwave Technol., vol. 18, pp. 1355-1363, Oct. 2000.

[5] R. Griffin, H. Salgado, P. Lane, and J. O'Reilly, "System capacity for millimeter-wave radio-over-fiber distribution employing an optically supported PLL," J. Lightwave Technol., vol. 17, pp. 2480-2487, Dec, 1999.

[6] G. H. Smith and D. Novak, "Broadband millimeter wave fiber-radio network incorporating remote up/down conversion," in Proc. IEEE Microwave Theory and Techniques Soc. Int. Symp., Baltimore, MD, 1998, pp. 1509-1512.

[7] C. Marra, A. Nirmalathas, C. Lim, M. Attygalle, D. Novak, B. Ashton, L. Poladian, W. Rowe, T. Wang, J. A. Besley, and L. Reekie, “A WDM fiber-radio system with improved optical spectral efficiency incorporating remote LO delivery and novel FBG optical filtering," in Proc. Optical Fiber Communication Conf. 2003, Altanta, GA, 2003, pp. 730-731.

[8] G. Smith, D. Novak, and Z. Ahmed, "Overcoming chromatic-dispersion effects in fiber-wireless systems incorporating external modulators," IEEE Trans. Microwave Theory Tech., vol. 45, pp. 1410-1415, Aug. 1997. 